\title{
Genetic polymorphism associated with infectious pulmonary diseases in siberian populations and among patients with community acquired pneumonia
}

\author{
Svetlana Vladimirovna Mikhailova \\ Lab. of human molecular genetics \\ ICG SB RAS \\ Novosibirsk, Russia \\ ORCID 0000-0002-0897-5473 \\ Irina Ivanovna Logvinenko \\ Lab. of preventive medicine \\ IIPM - a branch of the ICG SB RAS \\ Novosibirsk, Russia \\ ORCID 0000-0003-1348-0253
}

\author{
Liliya Valeryevna Shcherbakova \\ Laboratory of Clinical-Populational and \\ Prophylactic Studies on Internal and \\ Endocrine Diseases \\ IIPM - a branch of the ICG SB RAS \\ Novosibirsk, Russia \\ ORCID 0000-0001-9270-9188 \\ Mikhail Ivanovich Voevoda \\ Dep. of Human Molecular Genetics \\ ICG SB RAS \\ Novosibirsk, Russia \\ ORCID 0000-0001-9425-413X
}

\author{
Nadejda Ivanovna Logvinenko \\ Dep. of therapy, hematology and \\ transfusiology \\ Novosibirsk State Medical University \\ Novosibirsk, Russia \\ nadejda-logvinenko@yandex.ru
}

\begin{abstract}
Innate immune system is the first to respond to pathogen invasion; it activates adaptive immunity and regulates the intensity of inflammation. Two single nucleotide polymorphisms of the innate immunity genes: rs5743708 of the $T L R 2$ and rs8177374 of the TIRAP were shown to be associated with pneumonia and tuberculosis, but the data are contradictory in different ethnic groups. We assessed the prevalence of these variants in Caucasoid and Asian population samples, and among patients with community acquired pneumonia (CAP). Carriage of the rs5743708 A allele was found

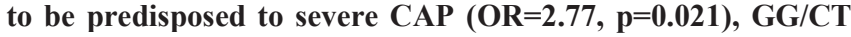

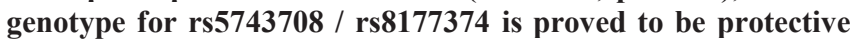
against it $(\mathrm{OR}=0.478, \mathrm{p}=0.022)$ in Caucasoid patients. Both Caucasoid and Asian studied populations were turn to be different from European and neighboring Asian populations on the genotypes prevalence. Differentiation of CAP by the causative pathogen could help to eliminate the currently observed contradictions between different research groups.
\end{abstract}

Keywords - genetic polymorphism, genetic predisposition, community acquired pneumonia, pulmonary tuberculosis, TLR2 gene, TIRAP gene

\section{Motivation and Aim}

Community acquired pneumonia (CAP) and pulmonary tuberculosis (TB) are the diseases characterized by high mortality; according to WHO, they are consistently among the ten leading causes of death in the world [1]. CAP is lower respiratory tract inflammatory disease caused mainly by viruses and bacteria. Improved diagnostic methods have recently shown that viruses, not Streptococcus pneumoniae, are the main cause of CAP. However severe pneumonia is more likely to develop in the case of combined infections and a seasonal increase in pneumonia coincides with seasonal outbreaks of influenza [2]. TB is chronic infectious disease, caused mainly by Mycobacterium tuberculosis. About a quarter of the world's population is infected with this bacterium and is at risk of developing the disease. Pathogenesis of these diseases is based on different mechanisms: excessive inflammation for CAP and the inhibition of acute immune response by $M$. tuberculosis for TB. Interestingly, for some genes of innate immunity in humans, an association with both CAP and pulmonary TB has been shown [3-6]. However, the data are contradictory in different racial and ethnic groups. In this work, we analyzed the frequencies for the rs5743708 (R753Q) of the TLR2 gene and rs8177374 (S180L) of the TIRAP gene in Caucasian and Asian samples of Russia, including long-lived people sample. In addition, we estimated the frequencies of these polymorphisms among patients with severe and non-severe CAP.

\section{Methods}

The adolescent sample was selected from the inhabitants of Novosibirsk city. Yakutian Caucasoid sample was collected in Tommot, Neryungri, Ust-Nera, and Yakutsk towns. These were people who lived in Yakutia for more than 16 years or were born there Asian sample consisted of Chukchis from Kanchalan village and Yakuts from Tommot, Neryungri, UstNera towns and Kylayy village. Long-lived people sample was collected in West Siberia. CAP patients sample was collected in offices of pulmonary hospitals of Novosibirsk and Yakutsk. CAP with various pathogenesis was regarded as severe if the CURB65 rating scale index was 4-5 points. All patients signed an informed consent to participate in the study. Genotyping was performed by PCR with analysis of restriction fragment length polymorphism.

\section{Results and Discussion}

Genotypes amount and frequencies for rs5743708 of the TLR2 gene and rs8177374 of the TIRAP gene are presented in Table1. 

PATIENTS

\begin{tabular}{|c|c|c|c|c|c|c|c|c|c|c|c|c|c|c|c|}
\hline \multirow[t]{2}{*}{ Samples \genotypes } & \multirow{2}{*}{$\begin{array}{c}\mathrm{n}^{\mathrm{a}} \\
\text { total }\end{array}$} & \multicolumn{2}{|c|}{ GG/CC } & \multicolumn{2}{|c|}{ GG/CT } & \multicolumn{2}{|c|}{ GG/TT } & \multicolumn{2}{|c|}{$\mathrm{AG} / \mathrm{CC}$} & \multicolumn{2}{|c|}{$\mathbf{A G} / \mathbf{C T}$} & \multicolumn{2}{|c|}{$\mathbf{A A} / \mathbf{C C}$} & \multicolumn{2}{|c|}{$\mathbf{A A} / \mathbf{C T}$} \\
\hline & & $n$ & $p^{b}$ & $n$ & $p$ & $n$ & $p$ & $n$ & $p$ & $n$ & $p$ & $n$ & $p$ & $n$ & $p$ \\
\hline Adolescents, Novosibirsk & 451 & 335 & 0.74 & 72 & 0.16 & 4 & 0.01 & 29 & 0.06 & 8 & 0.02 & 1 & 0.002 & 1 & 0.002 \\
\hline Caucasoids, Yakutia & 277 & 219 & 0.79 & 43 & 0.16 & 2 & 0.01 & 10 & 0.04 & 3 & 0.01 & 0 & 0 & 0 & 0 \\
\hline Long-lived peoples, West Siberia & 188 & 150 & 0.80 & 25 & 0.13 & 2 & 0.01 & 11 & 0.06 & 0 & 0 & 0 & 0 & 0 & 0 \\
\hline CAP total, Caucasoids & 406 & 307 & 0.76 & 71 & 0.18 & 2 & 0.004 & 22 & 0.05 & 3 & 0.01 & 0 & 0 & 0 & 0 \\
\hline Severe CAP & 120 & 93 & 0.78 & 13 & 0.11 & 1 & 0.01 & 11 & 0.09 & 2 & 0.02 & 0 & 0 & 0 & 0 \\
\hline Non- severe CAP & 286 & 215 & 0.75 & 58 & 0.20 & 1 & 0.004 & 11 & 0.04 & 1 & 0.004 & 0 & 0 & 0 & 0 \\
\hline Chukchis & 91 & 75 & 0.82 & 16 & 0.18 & 0 & 0 & 0 & 0 & 0 & 0 & 0 & 0 & 0 & 0 \\
\hline Yakuts & 120 & 111 & 0.93 & 6 & 0.05 & 0 & 0 & 3 & 0.03 & 0 & 0 & 0 & 0 & 0 & 0 \\
\hline Severe CAP, Yakuts & 33 & 28 & 0.85 & 3 & 0.09 & 1 & 0.03 & 1 & 0.03 & 0 & 0 & 0 & 0 & 0 & 0 \\
\hline
\end{tabular}

a number of people, b genotype frequency

The genotype distribution in all populations was in HardyWeinberg equilibrium. No differences in the alleles and genotypes were found between male and female. No significant difference was found between Russian adolescences and long-lived people samples. Apparently, the carriage of the studied alleles is not associated with a predisposition to longevity. There was significant difference in rs5743708 frequency between Novosibirsk adolescents and Caucasoid sample from Yakutsk $\left(\chi^{2}=4.647, p=0.032\right)$. No one allele or genotype was associated with CAP predisposition among Caucasoid in total. In case of pneumonia, carriage of the rs5743708A allele of the TLR2 gene predisposed to severe CAP (AG/CC $+\mathrm{AG} / \mathrm{CT}$ vs all $\mathrm{OR}=2.77,95 \%$ CI: $1.227-6.272, \mathrm{p}=0.021$ ); heterozygous genotype for rs8177374 of TIRAP gene in a combination with GG of TLR2 gene had protective effect against severe CAP (GG/CT vs all OR=0.478, 95\% CI: 0.251-0.909, $\mathrm{p}=0.022$ ). However, similar association was not observed in Yakuts, possibly due to a small sample size. In Russian, frequency of "protective" rs8177374T allele was lower than in European populations ( 0.10 vs 0.22$)$; in North Asians, it was higher than in neighboring Chinese populations (0.03-0.09 vs 0.01) [7].

Toll-like receptor 2, TLR2 heterodimerizes with TLR1 or TLR6 after recognition of pathogen-associated molecular patterns. TIRAP (TIR-domain containing adaptor protein) mediates downstream signaling of the TLRs resulting in the initiation of cytokine genes transcription. Both studied substitutions change proteins structure, but have opposite effect on the disease predisposition. This may be due to location of the rs5743708 in a binding site for TLR6 [8]. This substitution cans potentially increaseTLR2:TLR1 concurrent binding, while exactly TLR1 is responsible for NF-kB inflammatory signal cascade activation. The rs $8177374 \mathrm{~T}$ on the contrary, weakens signal transmission. The observed differences in the frequencies of variants of innate immunity genes in Asian populations can reflect the history of human impact with various pathogens during resettlement in Eurasia. "Pathogenic" for CAP and TB variant rs5743708A of the $T L R 2$ gene e.g. was shown to be protective against Lyme disease [9].

\section{Conclusions}

We found carriage of the rs5743708 A allele to be predisposed to severe CAP and $\mathrm{GG} / \mathrm{CT}$ genotype for rs5743708 / rs8177374 was protective against it in Caucasoid patients with pneumonia.

Differentiation of CAP by the causative pathogen could help to eliminate the currently observed contradictions between different research groups.

\section{ACKNOWLEDGMENT}

Supported by the SB RAS fundamental research program AAAA-A17-117072710029-7.

\section{REFERENCES}

[1] https://www.who.int/news-room/fact-sheets/detail/the-top-10-causesof-death

[2] J.A. McCullers, "The co-pathogenesis of influenza viruses with bacteria in the lung", Nat Rev Microbiol, vol 12, pp. 252-262, April 2014.

[3] I. Patarčić, A. Gelemanović, M. Kirin, I. Kolčić, E. Theodoratou, K.J. Baillie et al., "The role of host genetic factors in respiratory tract infectious diseases: systematic review, meta-analyses and field synopsis" Sci Rep, vol 5:16119, Nov 2015.

[4] T.V. Smelaya, O.B. Belopolskaya, S.V. Smirnova, A.N. Kuzovlev, V.V. Moroz, A.M/ Golubev, et al., "Genetic dissection of host immune response in pneumonia development and progression", Sci Rep, vol 6:35021, Oct 2016.

[5] L. Hu, H. Tao, X. Tao, X. Tang, and C. Xu, "TLR2 Arg753Gln gene polymorphism associated with tuberculosis susceptibility: An updated meta-analysis", Biomed Res Int, vol 2019:2628101, Jan 2019.

[6] C.C. Khor, S.J. Chapman, F.O. Vannberg, A. Dunne, C. Murphy, E.Y. Ling, et al, "A Mal functional variant is associated with protection against invasive pneumococcal disease, bacteremia, malaria and tuberculosis", Nat Genet, vol 39, pp.523-528, April 2007.

[7] https://www.ncbi.nlm.nih.gov/variation/tools/1000genomes

[8] Y. Xiong, C. Song, G.A. Snyder GA, E.J. Sundberg, and A.E. Medvedev, "R753Q polymorphism inhibits Toll-like receptor (TLR) 2 tyrosine phosphorylation, dimerization with TLR6, and recruitment of myeloid differentiation primary response protein 88 ", J Biol Chem, vol 287, pp. 38327-38337, Nov 2012.

[9] N.W. Schröder, I. Diterich, A. Zinke, J. Eckert, C. Draing, V. von Baehr et al., "Heterozygous Arg753Gln polymorphism of human TLR2 impairs immune activation by Borrelia burgdorferi and protects from late stage Lyme disease", J Immunol, vol 175, pp. 2534-2540, Aug 2005 . 